\title{
Comparing story reading and video watching as two distinct forms of vicarious contact: An experimental intervention among elementary school children
}

\author{
Veronica Margherita Cocco '* (D), Elisa Bisagno', \\ Gian Antonio Di Bernardo', Alessia Cadamuro', \\ Sara Debora Riboldi', Eleonora Crapolicchio', \\ Elena Trifiletti ${ }^{2}$, Sofia Stathi ${ }^{3}$ (D) and Loris Vezzali ${ }^{\prime}$ \\ 'University of Modena and Reggio Emilia, Italy \\ ${ }^{2}$ University of Verona, Italy \\ ${ }^{3}$ University of Greenwich, UK
}

\begin{abstract}
Research has shown that vicarious contact, that is observing an interaction between ingroup and outgroup members, can improve intergroup relations. Although vicarious contact has been operationalized in different ways, mainly via story reading or video watching, an experimental comparison of these different strategies is still missing. We conducted a school intervention with the aim of comparing the two most used forms of vicarious contact, namely story reading and video watching. Elementary schoolchildren without disabilities $(N=292)$ were assigned to one of three different conditions: reading a story; watching a video; control. In the two vicarious contact conditions, participants read or watched the story of a child with disability becoming friends with children without disabilities; in the control condition, participants only completed the dependent measures. Results revealed that, in general, both vicarious contact conditions were equally effective in improving outgroup attitudes and behavioural intentions. In addition, they operated with the same strength through the same underlying processes (IOS, ingroup norms). We discuss theoretical and practical implications in the context of vicarious contact as a prejudice-reduction intervention.
\end{abstract}

Despite that research on intergroup contact has shown that direct, face-to-face contact between groups can reduce prejudice (Hodson \& Hewstone, 2013; Pettigrew \& Tropp, 2006), the implementation of direct contact is often impractical. Research has, however, demonstrated that indirect contact, that is contact that is not face to face, can also help improve intergroup relations. Among the different forms of indirect contact strategies that can improve attitudes, in this research we focus on vicarious contact, that is the observation of interactions between ingroup and outgroup members. This form of contact is distinct from extended contact, that is knowing about positive intergroup interactions

*Correspondence should be addressed to Veronica Margherita Cocco, Università di Modena e Reggio Emilia, Viale Del Pozzo 7I, Modena 2 I I 24, Italy (email: veronicamargheritacocco@gmail.com). 
(Brown \& Paterson, 2016; Dovidio, Eller, \& Hewstone, 2011; Vezzali, Hewstone, Capozza, Giovannini, \& Wölfer, 2014; Wright, Aron, McLaughlin-Volpe, \& Ropp, 1997).

There is now a substantial amount of research showing that vicarious contact improves outgroup attitudes (Brown \& Paterson, 2016; Schrijvers, Jannsen, Fialho, \& Rijlaarsdam, 2019; Turner, Hewstone, Voci, Paolini, \& Christ, 2007; Vezzali et al., 2014). The operationalization of this indirect contact strategy has been rather heterogeneous. In particular, vicarious contact has been mainly operationalized by means of written stories (Cameron \& Rutland, 2006) or videos depicting intergroup interactions (Mazziotta, Mummendey, \& Wright, 2011). However, these two operationalizations, despite both being identified as vicarious contact strategies, might have differential effects and operate through distinct processes. In other words, it is currently not clear whether the positive effects of vicarious contact depend on the medium through which it is expressed. Understanding these similarities and differences is especially important when conducting prejudice-reduction interventions in educational contexts (where several vicarious contact studies have been conducted), since it can allow to better calibrate interventions and their effects. The aim of this article is precisely to explore the similarities or differences of these two vicarious contact strategies in the context of a prejudicereduction intervention conducted at school. Specifically, we experimentally manipulated and compared vicarious contact via a written story or a video with elementary schoolchildren without disabilities, the target being children with disabilities. In addition, we explored and compared the underlying processes between the two strategies.

\section{Vicarious contact}

Mazziotta et al. (2011) conducted a classic and often-cited study on vicarious contact. In Study 1 , in the experimental condition, German participants watched video clips carefully prepared by the authors, showing a positive interaction between a German and a Chinese actor. Results revealed that, compared to a control condition where participants watched intragroup interactions, participants in the experimental condition displayed more positive attitudes towards and intention to have contact with Chinese people. In the second study, results were replicated by including a further control condition where the outgroup member did not interact with other people (for other experimental studies using videos, see, e.g., Castelli et al., 2012, Study 1; Mallett \& Wilson, 2010; Nguyen, Chen, \& O'Reilly, 2012; West \& Turner, 2014).

The research presented above has largely made use of videos to implement vicarious contact and has been mainly conducted in the laboratory. Research in educational contexts has also taken advantage of vicarious contact strategies, likely due to its ease of operationalization compared to direct contact. In this case, however, vicarious contact has been generally implemented with the use of ad hoc created written stories read to participants by researchers (for exceptions, see Gómez \& Huici, 2008; Vezzali, Di Bernardo, Stathi, Visintin, \& Hewstone, 2019). One example of such method is a series of interventions conducted by Cameron and colleagues (Cameron \& Rutland, 2006; Cameron, Rutland, \& Brown, 2007; Cameron, Rutland, Brown, \& Douch, 2006; Cameron, Rutland, Hossain, \& Petley, 2011). In these studies, experimenters read and discussed stories of cross-group friendships (inspired by children's books, and accurately prepared by experimenters) to small groups of elementary schoolchildren over multiple sessions. There are now several examples of studies conducted in educational contexts showing the effectiveness of story reading, (Aronson et al., 2016; Greenwood et al., 2016; Husnu, Mertan, \& Cicek, 2018; Liebkind, Mähönen, Solares, Solheim, \& Jasinskaja-Lathi, 2014; 
Liebkind, Mäkinen, Jasinskaja-Lahti, Renvik, \& Solheim, 2019; Liebkind \& McAlister, 1999; Mäkinen, Liebkind, Jasinskaja-Lahti, \& Renvik, 2019; McKeown, Williams, \& Pauker, 2017; for a vicarious contact study using story reading outside an educational context, see Cernat, 2011; for reviews, see Cameron \& Turner, 2017; Di Bernardo, Vezzali, Stathi, Cadamuro, \& Cortesi, 2017).

\section{Mediating mechanisms}

Wright et al. (1997) originally proposed four mechanisms that drive the effects of extended or vicarious contact. The first is intergroup anxiety: Anxiety should be lower when learning or observing positive intergroup interactions than when being directly involved in contact. Second, observing ingroup and outgroup members in close contact should favour the perception that they constitute a cognitive unit (Sedikides, Olsen, \& Reis, 1993), therefore increasing psychological closeness with the outgroup, a mechanism known as inclusion of the other in the self (IOS; Aron, Aron, \& Smollan, 1992). Third and fourth, while individuals can be afraid of rejection by ingroup (Eller, Gómez, Vázquez, \& Fernández, 2017) and outgroup members (Shelton \& Richeson, 2005), observing them having contact can increase perceptions that ingroup and outgroup members are actually willing to interact, therefore shaping positive norms that both groups are favourable to contact. Although evidence for these mechanisms is larger for extended contact (cf. Vezzali et al., 2014), there is also evidence that vicarious contact improves outgroup attitudes because it reduces intergroup anxiety (Cernat, 2011), fosters perceptions of procontact ingroup and outgroup norms (Cameron et al., 2011), and enhances IOS (Vezzali, Stathi, \& Giovannini, 2012).

We also considered other potential mediating processes that have been shown to be relevant in the more general contact literature. Specifically, we focused on cognitive (i.e., perspective-taking) and affective empathy. Research has widely shown that direct (Pettigrew \& Tropp, 2008) and extended contact (Birtel, Vezzali, \& Stathi, 2017) operate via increased perspective-taking and affective empathy towards the outgroup. Although we are not aware of any vicarious contact studies testing perspective-taking and affective empathy as mediators, in line with the larger direct and extended contact literature, we expect that both would mediate the effects of vicarious contact (for evidence of mediation of media contact effects via television, radio, and newspapers - including simple exposure to the outgroup in addition to exposure to intergroup interactions - see Pagotto \& Voci, 2013).

\section{The present research}

The aim of this study was to test whether different operationalizations of vicarious contact, namely vicarious contact via story reading or video watching, would have different effects on a range of variables related to intergroup relations. We also tested whether the two forms would act through different underlying processes and the relative strength of them. A further aim was to provide the first test of the four mediating processes proposed by Wright et al. (1997) for a vicarious contact study.

To test the hypotheses, we conducted a prejudice-reduction intervention with elementary schoolchildren in school, a context where the effects of vicarious contact can be particularly useful. Participants were children without disabilities; the target outgroup was represented by children with disabilities. In the two vicarious contact conditions, we created an ad hoc story of a child with disability who, after experiencing initial difficulties 
in interactions with his schoolmates without disabilities, is accepted and becomes part of their friendship network. By maintaining the content stable, the story was presented to children in written or video format. After being presented with the story, children completed a questionnaire with the dependent measures. In the control condition, children were simply administered the questionnaire. This way, by maintaining the content stable, it was possible to test whether the medium (written story vs. video) influences the effectiveness of vicarious contact.

Based on previous literature, we expect effects of both story reading and video watching on outgroup attitudes. Research in the laboratory has generally made use of videos whereas research in the field has largely relied on written stories, using experimental procedures varying on a number of factors (e.g., number of written stories/videos presented, number of sessions, delay of dependent variables assessment). Findings for the two vicarious contact operationalizations are thus hardly comparable. Therefore, we cannot make specific predictions on how they will differentially affect outgroup variables, based on existing empirical evidence.

Although theorizing from a vicarious contact perspective, we can tangentially draw upon distinct theoretical approaches too. Based on the persuasion literature, the elaboration likelihood model (ELM; Petty \& Cacioppo, 1986) would predict that results for story reading and video watching will not differ. The ELM distinguishes a central from a peripheral route of persuasion. When individuals are able to process the message and are motivated to do it, they follow the central route and pay attention to the content of the message. Instead, when ability and motivation are lacking, they are more likely to follow the peripheral route, paying attention to peripheral cues, like visual factors. In this study, we have created a story that reflects a scenario that resonates with children's lives, making it relevant and interesting, and thus increasing their motivation to attend to its cues. We will present the story in small groups to allow them to pay attention to the story. Participants should therefore be more likely to follow the central route, paying attention to the content of the story (regardless of whether this is presented in a video or in written format) rather than to other peripheral elements (that may slightly differ across mediums used).

In addition, following a media framing approach, attitudes can change because the media make specific aspects and cognitions already present in the perceiver salient and reinforce them over time by increasing their importance, leading to attitude change (Price, Tewksbury, \& Powers, 1997). This approach places importance on how information is framed, that is greater prominence of some attributes will activate consistent cognitions, reinforcing them (Nelson, Oxley, \& Clawson, 1997; Scheufele, 2004). In the case of our research, making patterns of intergroup friendships salient should increase the importance attributed to such cognitions, leading to more positive outgroup attitudes. Since we aim to maintain consistency in the content reflected by the story, independently from the medium with which this is presented, we do not expect differential effects between experimental conditions.

We can also make a tentative hypothesis by relying on social cognitive theory (Bandura, 1997, 2002). According to the process of abstract modelling posited by this theory, individuals can learn patterns of attitudes and behaviours from the observation of relevant others, including models portrayed in the media. In addition, the process of symbolic interaction allows individuals to acquire cognitive and affective responses learnt from the observation of characters (Bandura, 1999). Social cognitive theory therefore highlights the importance of the observation of media characters. In the case of our study, the videos allow to actually observe characters interacting, making the process of social 
inclusion of the child with disability more salient. Based on these considerations, we may expect a greater effect of video watching rather than story reading.

With respect to underlying processes, we included the four main mechanisms identified by the extended and vicarious contact literature, that is intergroup anxiety, IOS, ingroup and outgroup norms. The rationale is that, this being the first study to compare two qualitatively different operationalizations of vicarious contact, it would be important to understand whether their effects are differently mediated by the most relevant mechanisms identified by research. In addition, based on the larger literature, we included perspective-taking and affective empathy that have been repeatedly shown to mediate direct and extended contact effects (Pettigrew \& Tropp, 2008; Vezzali et al., 2014). Although in this case too we are not able to make specific predictions, we can tentatively predict no differences between the two vicarious contact conditions; however, based on social cognitive theory, we might also predict that the actual observation (video watching) of behaviour produces stronger indirect effects via the hypothesized mediators compared to story reading (Bandura, 2002).

We selected a wide range of outcome variables, because of their relevance in the contact literature. As an outcome measure, with the aim of providing a solid comparison with previous studies, we included a measure of outgroup attitudes often used in child samples (Multiple-Response Racial Attitude measure; Aboud, 2003; Doyle \& Aboud, 1995). Since the intervention tested aimed to foster social inclusion, we also included a measure of social distance, hypothesizing that social distance should be lower after the intervention. Furthermore, given the relevance of behavioural intentions in predicting behaviour (Godin \& Kok, 1996), we included two different types of behavioural intentions: intentions to have contact with the outgroup in the future and intentions to help the outgroup. The first (contact intentions) is often assessed in vicarious contact studies conducted with children (e.g., Cameron \& Rutland, 2006), allowing us to compare results with previous findings, and assessing an outcome directly relevant to our manipulation (i.e., measuring the intention to spend time with the outgroup as a consequence of an intervention focused on social inclusion). With respect to helping intentions, they have rarely been used in the vicarious or direct and indirect contact literature (but see recent studies conducted with child and adolescent samples, e.g., Taylor \& Glen, 2020; Vezzali, Birtel, et al., 2019; Vezzali et al., 2019). However, we believe this variable is especially relevant in this research, as it can indicate whether the intervention was effective in promoting prosocial actions.

\section{Method}

\section{Participants}

Participants were 296 non-disabled elementary schoolchildren from a primary school located in Northern Italy. We excluded four participants because of difficulties in filling the final questionnaire. The final sample is therefore composed of 292 children (152 males, 140 females) from first to fourth grade (age therefore ranged roughly from 6 to 9 years old, although information on exact age is not available). Participants were allocated to one of the three conditions: video $(N=107)$, reading $(N=86)$, control $(N=99)$. Because of organizational reasons within the schools, for each grade, classes rather than individual participants were allocated to the three conditions. Participants' parents provided informed consent for their children; we also collected verbal children's consent before their participation. 
Sample size was determined according to school availability. Specifically, we calculated a range of participants needed for running a one-way ANOVA with three groups (i.e., video, reading, and control) allowing a power of .80 to detect a medium $\left(f^{2}=0.25\right)$ to small $\left(f^{2}=0.15\right)$ effect size. Thus, the final aim was to obtain a pool of participants included between 432 (i.e., about 144 respondents per group) and 156 children (i.e., about 52 respondents per group). This sample size range was also sufficient to test a multiple regression with up to eight predictors (two dummies and six mediators, see predictions), allowing a power of .80 for detecting a medium $\left(f^{2}=0.15\right)$ to small $\left(f^{2}=0.04\right)$ effect size.

\section{Procedure}

Researchers conducting the intervention were students enrolled in educational academic courses at a northern Italian university. All researchers were trained by the second and last authors of the present article. Before running the study, we created an ad hoc story featuring a disabled child in a wheelchair, in line with previous stories used in educational interventions based on vicarious contact (e.g., Cameron \& Rutland, 2006). In this story, the disabled child wishes to play with classmates, but since nobody invites him, he spends his time playing alone, listening to music, drawing, or imagining fantastic stories. When he asks his desk companion to play together, she answers with some embarrassment that he would not be able to play the games they are playing. When he replies that instead he could do that, she and other three friends of her invite him to play together, discovering that they could easily play together, simply by engaging in the games a bit differently. The group of children without disabilities changes its mind towards peers with disabilities, also stating that they are sorry for their past behaviour. Everybody is then very helpful towards the child with disability, and this newly established friendship persists over the years, leading to a change of attitudes and behaviours of characters without disabilities towards children with disabilities as a whole. We also created a video-cartoon with free online programs (Toontastic for realizing the cartoon; OpenShot for video editing; Audacity for the audio). ${ }^{1}$

We took several steps to ensure that written story and video were as equivalent as possible and that the same elements were emphasized in both conditions. First, the written story included images of the main characters, which were the same used in the videos. Second, details such as names of characters, settings, and actions were the same, and the order of presentation of the different scenes was identical. Third, in line with previous vicarious contact studies (e.g., Cameron \& Rutland, 2006), the stories in the reading condition were presented verbally by the researcher (see below), minimizing effects due to differential literacy skills of children. Finally, the time of presentation for both the written story and the video was the same, approximately $10 \mathrm{~min}$.

The intervention was administered in Italian. Participants were randomly allocated (at the class level) to the three conditions. In the reading condition, in each class, in order to enhance children's attention, participants were divided into small groups of approximately 3-6 members. The experimenter read the story and then discussed it briefly with the children. The watching condition was identical to the reading condition. In this case, however, children were shown the video by means of tablets or smart phones. In both conditions, at the end of the session, children were administered a face-to-face

\footnotetext{
I The written story and the video are available upon request to the first author.
} 
questionnaire in Italian language. Researchers that administered the intervention were different from those who administered the questionnaire, in order to address possible demand characteristics. Participants in the control condition were only administered the questionnaire. Participants were finally thanked and debriefed.

\section{Measures}

Unless otherwise indicated, a 5-point Likert scale ranging from 1 (absolutely not) to 5 (absolutely yes) was used; 3 was the neutral point (maybe not, maybe yes).

\section{Manipulation check}

In order to test whether the story presented in written text or in video were comparable in terms of likeability or understanding, participants were asked the following four questions: 'Did you like the story?'; 'Was the story beautiful?'; 'Did you understand the story?'; 'Was the story easy to understand?'

Inclusion of the other in the self

IOS was assessed with one item (Aron et al., 1992). Children were asked to imagine meeting an unknown child with disability from their school in the park. The possible answers were represented by five pairs of overlapping circles varying in their degree of overlap between the self as one circle and the child with disability as the other circle. Participants were asked to indicate the pair of circles that best described their closeness to this child. Higher scores indicated greater closeness (for a similar measure, see, e.g., Cameron et al., 2006; Vezzali et al., 2019).

\section{Intergroup anxiety}

Participants answered three items, adapted from items used to assess intergroup anxiety in research on intergroup contact (cf. Lolliot et al., 2015; Stephan \& Stephan, 1985) and adapted to a child sample. In particular, children were asked whether they would feel anxious, nervous, tense, at the prospect of meeting an unknown child with disability from their school at the park. The three items were averaged in an index of intergroup anxiety $(\alpha=.67)$.

\section{Ingroup and outgroup norms}

We used three items for ingroup and three items for outgroup norms, adapted from Turner, Hewstone, Voci, and Vonofakou (2008), and from Vezzali, Stathi, Giovannini, Capozza, and Visintin (2015). Participants were first asked to imagine that a child with disability would arrive to their class and then asked whether they think that their schoolmates would like the child, would like to play, would like to be friends with this child (ingroup norms), and whether they think this child with disability would like the schoolmates, would like to play, would like to be friends with his/her schoolmates (outgroup norms). Items were combined in two indices of ingroup $(\alpha=.71)$ and outgroup norms $(\alpha=.71)$, respectively. 


\section{Perspective-taking}

We used two items from Vezzali, Birtel, et al. (2019; see also Capozza, Trifiletti, Vezzali, \& Favara, 2013): 'Do you see things as disabled children in wheelchair see things?'; 'Do you think in the same way as disabled children in wheelchair think?' The two items were merged to form a composite index of perspective-taking $(r=.36, p<.001)$.

\section{Affective empathy}

Affective empathy was assessed with the following two items from Vezzali, Birtel, et al. (2019; see also Capozza et al., 2013): 'Do you understand the emotions felt by disabled children in wheelchair?'; 'Do you feel the same emotions felt by disabled children in wheelchair?' Items were combined in a single index of affective empathy $(r=.20$, $p=.001)$.

\section{Outgroup attitudes}

We measured intergroup attitudes with the Multiple-Response Racial Attitude measure (Aboud, 2003; Doyle \& Aboud, 1995), adapted for child use in the Italian context by Birtel et al. (2019) and for use with children with disability as the outgroup. Participants were randomly presented eight traits, both positive (good, nice, likeable, clean) and negative (unpleasant, bad, ugly, dirty). For each trait, the task was to decide whether to assign it to an ingroup member (a drawing of a same-sex child without disability), to an outgroup member (a drawing of a same-sex child in a wheelchair), to both an ingroup and an outgroup member (the two drawings of the same-sex children with and without disabilities), or to nobody. We computed the number of positive and negative traits assigned to outgroup members: A trait was calculated as assigned to the outgroup when the participants assigned it to the outgroup specifically or to both groups (two out of the four options given to participants; see above). We then calculated the difference between positive and negative traits assigned to the outgroup to obtain a single index of outgroup attitudes, with higher scores denoting more positive outgroup attitudes (range: from -4 to +4 ).

\section{Social distance}

Three items were used, adapted from Esses and Dovidio (2002) and used with children in the Italian context by Vezzali et al. (2018). In particular, participants were asked whether they would be happy to have an unknown child with disability as a neighbour, friend, schoolmate. After reverse-scoring the items, they were combined in an index of social distance $(\alpha=.75)$, with greater scores denoting more social distance.

\section{Contact intentions}

Participants were presented with three items. Specifically, they were asked whether they would be happy to meet, play, go and have an ice cream with an unknown child with disability they met at the park (see Cameron \& Rutland, 2006; Vezzali, Birtel, et al., 2019). The three items were averaged in a single measure of contact intentions $(\alpha=.77)$.

\section{Helping intentions}

Three items were used, adapted from Vezzali, Stathi, Crisp, et al. (2015, Study 1), for example, 'Imagine that a child with disability has got lost at school and asks to help him/ 
her to find his/her class. Would you help him/her?' We averaged ratings in a composite measure $(\alpha=.75)$, with higher scores reflect stronger intention to help children with disability. $^{2}$

We also included number of friends with disability as a control variable. Participants were provided with an open question where they were asked to indicate the names of their friends with disability (the score therefore corresponds to the number of nominated friends).

\section{Results}

\section{Manipulation check}

Results of comparisons on the four manipulation check items between reading and watching condition revealed no significant differences. Specifically, in both conditions, children liked the story $\left(M_{\text {reading }}=4.69, S D_{\text {reading }}=0.67 ; M_{\text {watching }}=4.69, S D_{\text {watch- }}\right.$ ing $=0.76)$, stated that the story was nice $\left(M\right.$ reading $=4.67, S D_{\text {reading }}=0.60 ; M_{\text {watching }}=4.64$, $\left.S D_{\text {watching }}=0.73\right)$, understood the story $\left(M_{\text {reading }}=4.22, S D_{\text {reading }}=0.92 ; M_{\text {watch- }}\right.$ ing $=4.04, S D$ watching $=1.02)$, and stated that the story was easy to understand $\left(M_{\text {read- }}\right.$ ing $=3.85$, sDreading $\left.=1.24 ; M_{\text {watching }}=3.78, S D_{\text {watching }}=1.13\right), t \mathrm{~s}<1.30, p \mathrm{~s}>.196$. Based on these findings, we can conclude that any eventual differences between conditions can be attributed to differences between types of vicarious contact, rather than to the likeability or understanding of the story between the two vicarious contact conditions.

\section{Main analyses}

Means, standard deviations, and differences between conditions are presented in Table 1 (dependent variables) and Table 2 (hypothesized mediators); correlations are shown in Table 3. As can be seen in Table 1, the intervention had effects on two dependent variables. In particular, outgroup attitudes and helping intentions were higher in the watching compared with the control condition. In addition, outgroup attitudes were more positive in the reading than in the control condition. Although the effect did not reach statistical significance, we also found a tendency for social distance to be lower in the watching than in the control condition. ${ }^{3}$

With respect to hypothesized mediators, we obtained effects for IOS, ingroup norms, and affective empathy. As can be seen in Table 2, replicating effects obtained for the dependent variables, the scores of these variables were higher in the watching than in the control condition. Similar results were obtained for the reading condition, where IOS and affective empathy were more positive than in the control condition. No differences emerged between watching and reading conditions.

\footnotetext{
${ }^{2}$ The questionnaire also included two additional measures. One was a measure of one-group identity that we included with exploratory purposes. Results revealed that the effect of condition was non-significant, $F(2,289)=I .76, p=.173, \eta_{\mathrm{p}}^{2}=.01$. The second was a measure of resource allocation. However, since there were mistakes and differences in how this was administered to participants, we decided to exclude it from analyses.

${ }^{3}$ We also computed an index of ingroup attitudes and a measure of attitudinal bias, by calculating the difference between ingroup and outgroup attitudes. Results for ingroup attitudes revealed a marginal effect of condition, $F(2,289)=2.40, p=.092$, $\eta_{\mathrm{p}}^{2}=.02$. Post-hoc comparisons revealed that ingroup attitudes were marginally more positive $(\mathrm{ps}<.07)$ in the reading $(M=2.64)$ and watching $(M=2.58)$ conditions than in the control condition $(M=2.12)$. The ANOVA was non-significant for the measure of attitudinal bias, $F<I$.
} 
Table I. Means and standard deviations (in parentheses) of dependent variables in the three experimental conditions, and results of ANOVAs $(N=292)$

\begin{tabular}{llllll}
\hline & \multicolumn{3}{l}{ Condition } & & \\
\cline { 2 - 5 } Measure & Reading $(N=86)$ & Watching $(N=107)$ & Control $(N=99)$ & $F(2,289)$ & $\eta_{\mathrm{p}}^{2}$ \\
\hline Outgroup attitudes & $2.91 \mathrm{a}(\mathrm{I} .49)$ & $2.96 \mathrm{a}(\mathrm{I} .52)$ & $2.39 \mathrm{~b}(\mathrm{I} .92)$ & $3.55^{*}$ & .02 \\
Social distance & $1.90(\mathrm{I} .03)$ & $1.63(0.79)$ & $1.86(0.96)$ & $2.49^{\dagger}$ & .02 \\
Contact intentions & $4.34(0.95)$ & $4.48(0.70)$ & $4.30(0.83)$ & 1.28 & .01 \\
Helping intentions & $4.75 \mathrm{ab}(0.54)$ & $4.84 \mathrm{~b}(0.36)$ & $4.66 \mathrm{a}(0.60)$ & $3.24^{*}$ & .02 \\
\hline
\end{tabular}

Note. The response scale ranges from I to 5 , with the exception of the measure of outgroup attitudes, which ranges from -4 to +4 . Different letters on the same row indicate that the means are significantly different, $p<.05$. In the case of the comparison between watching and control condition for the measure of social distance, the difference was marginally significant, $p<.08$.

${ }^{\dagger} p<.10 ; * p<.05 ; * * p<.01 ; * * * p \leq .001$.

Table 2. Means and standard deviations (in parentheses) of hypothesized mediators in the three experimental conditions, and results of ANOVAs $(N=292)$

\begin{tabular}{|c|c|c|c|c|c|}
\hline \multirow[b]{2}{*}{ Measure } & \multicolumn{3}{|l|}{ Condition } & \multirow[b]{2}{*}{$F(2,289)$} & \multirow[b]{2}{*}{$\eta_{\mathrm{p}}^{2}$} \\
\hline & Reading $(N=86)$ & Watching $(N=107)$ & Control $(N=99)$ & & \\
\hline Intergroup anxiety & $2.36(1.18)$ & $2.34(1.06)$ & $2.5 \mathrm{I}(\mathrm{I} .12)$ & 0.67 & .00 \\
\hline IOS & $4.28 \mathrm{a}(1.17)$ & $4.22 \mathrm{a}(1.1 \mathrm{I})$ & $3.68 \mathrm{~b}(1.34)$ & $7.25 * * *$ & .05 \\
\hline Ingroup norms & 4.06ab (1.0I) & $4.12 \mathrm{a}(0.93)$ & $3.8 \mathrm{lb}(0.92)$ & $2.93^{\dagger}$ & .02 \\
\hline Outgroup norms & $4.33(0.77)$ & $4.40(0.72)$ & $4.18(0.88)$ & 2.63 & .01 \\
\hline Perspective-taking & $3.22(1.40)$ & $3.22(1.24)$ & $3.05(1.21)$ & 0.56 & .00 \\
\hline Affective empathy & $3.60 \mathrm{a}(1.14)$ & $3.56 \mathrm{a}(1.1 \mathrm{I})$ & $3.20 \mathrm{~b}(1.25)$ & $3.45^{*}$ & .02 \\
\hline
\end{tabular}

Note. IOS $=$ Inclusion of the other in the self. For all measures, the response scale ranges from I to 5 . Different letters on the same row indicate that the means are significantly different, $p<.05$. In the case of the comparison between reading and control condition for the measure of ingroup norms, the difference was marginally significant, $p<.08$.

${ }^{\dagger} p<.10 ; * p<.05 ; * * p<.01 ; * * * \leq .001$.

\section{Mediation processes}

The hypothesized mediation models were tested by running a series of regressions (one for each dependent variable) using PROCESS macro for SPSS (Model 4; Hayes, 2013). Independent variables were represented by two dummy variables. For the first dummy variable, 1 was assigned to the watching condition, while 0 indicated the reading and control conditions; for the second dummy variable, reading condition (1) was compared with watching and control conditions (0). Mediators were represented by affective empathy, ingroup norms, and IOS (conditions did not have an effect on the other hypothesized mediators; cf. Table 2), while outgroup attitudes, social distance, helping, and contact intentions served as dependent variables.

As can be seen in Table 4, both interventions (i.e., watching and reading) positively affected all the dependent variables via increased IOS. In addition, the effects of reading and watching conditions were mediated by ingroup norms, with one exception: Ingroup norms did not mediate the effect of reading on social distance. Finally, affective empathy mediated the effects of both intervention conditions on outgroup attitudes and social distance, but not on contact and helping intentions. 


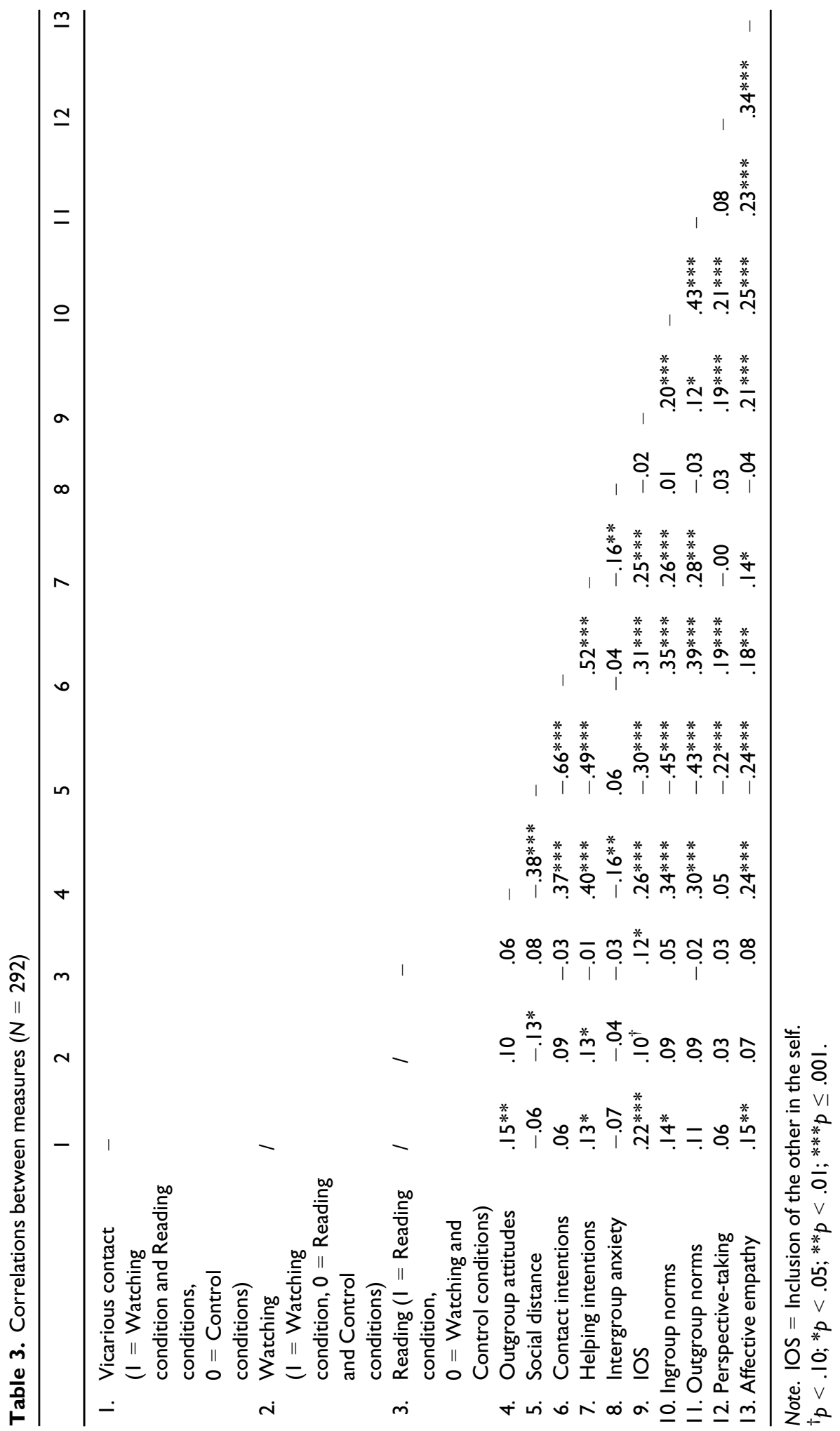


In order to further investigate the differential effects of the two types of vicarious contact, we compared the mean bootstrap estimates using the method proposed by Cummings (2009); that is, a coefficient is significantly different from another if the respective confidence intervals overlap by less than $50 \%$. From Table 4 , considering the mediation effects, it can be noted that all the confidence intervals related with the watching intervention largely coincide with the ones related to the reading intervention, indicating that no difference emerged between the two sets of contact strategies in terms of strength of underlying processes. ${ }^{4}$

\section{Additional analyses}

In contrast with research on extended contact, where various studies have simultaneously tested the mediating role of the four mechanisms proposed by Wright et al. (1997), that is intergroup anxiety, IOS, ingroup, and outgroup norms (Gómez, Tropp, \& Fernández, 2011; Turner et al., 2008), no vicarious contact study to date has tested the four processes simultaneously. An additional aim of this research was to provide the first such test. A series of multiple regressions (PROCESS, Model 4, see the paragraph above; Hayes, 2013) were conducted. As independent variable, a new dummy representing vicarious contact was created, namely, 1 was assigned to both experimental conditions and 0 was assigned to the control condition; mediators were represented by ingroup and outgroup norms, intergroup anxiety, IOS; dependent variables were the same included in the previous models (cf. Table 1).

From results, it emerged that vicarious contact significantly affected IOS $(B=.56$, $S E=.15, p<.001)$ and ingroup norms $(B=.28, S E=.12, p<.05)$ that, in turn were related to outgroup attitudes $(B=.24, S E=.07, p<.01$ for IOS; $B=.43, S E=.10$, $p<.001$ for ingroup norms), social distance $(B=-.17, S E=.04, p<.001$ for IOS; $B=-.31, S E=.05, p<.001$ for ingroup norms), and contact intentions $(B=.17$, $S E=.04, p<.001$ for IOS; $B=.17, S E=.04, p<.001$ for ingroup norms). Regarding helping intentions, it was only positively predicted by $\operatorname{IOS}(B=.08, S E=.02, p<.001)$. All the indirect paths reported above turned out being significant: vicarious contact $\rightarrow$ IOS $\rightarrow$ outgroup attitudes (effect $=.36, S E=.12,95 \%$ CI $[0.1689,0.6371]$ ); vicarious contact $\rightarrow$ ingroup norms $\rightarrow$ outgroup attitudes (effect $=.12, S E=.06,95 \%$ CI [0.0242, $0.3050]$ ); vicarious contact $\rightarrow$ IOS $\rightarrow$ social distance (effect $=-.10, S E=.04,95 \% \mathrm{CI}$ $[-0.1955,-0.0368]$ ); vicarious contact $\rightarrow$ ingroup norms $\rightarrow$ social distance (effect $=-.09, S E=0.05,95 \%$ CI $[-0.2021,-0.0183])$; vicarious contact $\rightarrow$ IOS $\rightarrow$ contact intentions (effect $=.10, S E=.04,95 \% \mathrm{CI}[0.0360,0.1951])$; vicarious contact $\rightarrow$ ingroup norms $\rightarrow$ contact intentions (effect $=.05, S E=.03,95 \%$ CI [0.0088, 0.1297]); vicarious contact $\rightarrow$ IOS $\rightarrow$ helping intentions (effect $=.04, S E=0.02,95 \%$ CI $[0.0128,0.0994])$.

\section{Discussion}

In this study, we aimed to compare two different forms of vicarious contact, namely story reading and video watching in the context of a naturalistic intervention, in order to

\footnotetext{
${ }^{4}$ The pattern of results did not change when including sex, grade (which was used as a replacement for age, since age was not accurately reported by a large part of the sample), and number of friends as covariates, with three exceptions: For the measure of social distance, the comparison between watching and reading conditions became marginal; for the measure of ingroup norms, the comparison between reading and control conditions became significant (it was marginal); concerning mediation effects, the effect of watching on social distance via affective empathy became non-significant.
} 


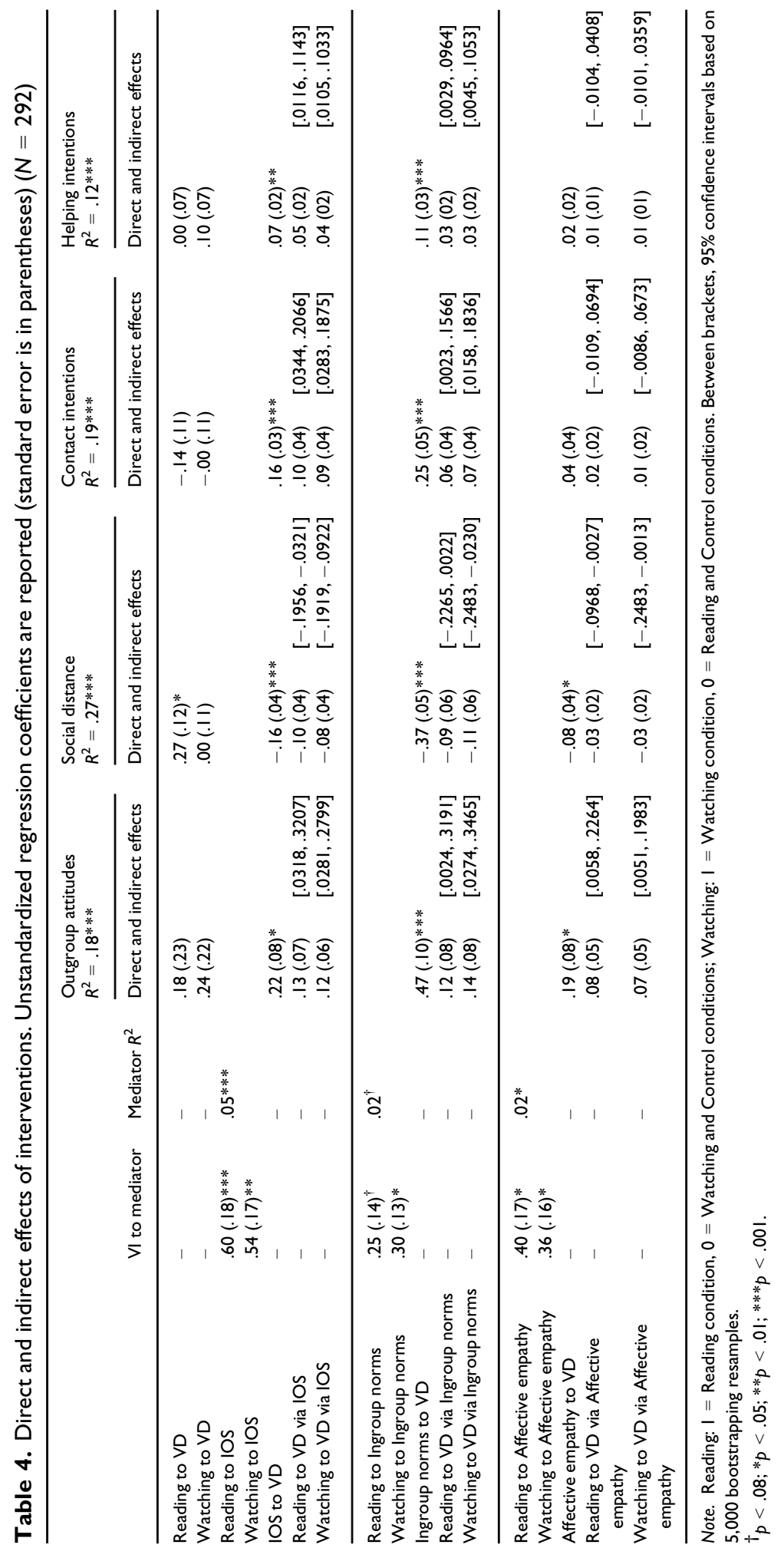


understand whether effects depend on the specific medium (written stories or videos). Research has in fact tested these two strategies separately and mostly in different settings, leaving questions regarding the role of reading vs. watching independently from the content unanswered.

First of all, in line with the literature (Vezzali et al., 2014), vicarious contact was effective at improving outgroup attitudes. It also represents the first test of vicarious contact where all four mediators identified by Wright et al. (1997) are simultaneously tested (see the section of Additional analyses). More relevant to the present aims, the general pattern of results points to a similar effectiveness of reading and watching. In fact, reading and watching conditions had similar effects on dependent variables (although, for the measure of helping intentions, the watching but not the reading condition differed from the control condition). Similar effects have been obtained with respect to mediators, where both conditions had significant effects on three out of the six tested mediators (although for the measure of ingroup norms, the effects of the reading condition were only marginally different from the control condition), without differences between them.

These considerations are reinforced by the examination of underlying processes. In particular, the effects of both conditions were mediated by IOS (all variables), ingroup norms (all variables, except the indirect effect of the reading condition on social distance), and affective empathy (limited to outgroup attitudes and social distance). Importantly, the strength of indirect effects did not differ between the two conditions, showing that not only main effects, but also mediating processes do not differ depending on how the content of vicarious contact is presented.

These findings are consistent with hypotheses we derived from persuasion research. We argue that participants may have followed the central route posited by the ELM (Petty \& Cacioppo, 1986), focusing on the content of the story. This may have been facilitated by the fact that the story was created to target this group of participants, ensuring that they were able to process it (as confirmed by the teachers who approved of the materials and by the manipulation checks) and that the story was relevant to their life. Possibly, differences between means of communication are more likely to emerge when individuals engage with the story superficially, therefore focusing on peripheral elements. Future studies should investigate the degree of elaboration, to understand whether this may be a moderator of the effects.

One might speculate that the videos, providing a richer visual environment, would favour the vividness of mental imagery related to the story. To the extent that the degree of vividness can influence attitude change and associated intentions and behaviour (Husnu \& Crisp, 2010), the effects should have been stronger in the video than in the reading condition. This would also be consistent with the tentative hypothesis we made based on social cognitive theory (Bandura, 2002). The absence of differences may, however, indicate that both experimental conditions produced equally vivid images. However, this hypothesis is only speculative, since we did not assess vividness of mental images as a function of condition.

Although linguistic and visual information is perceived and processed differently (Kress \& van Leeuwen, 2010), information in the media is generally conveyed in multimodal contexts, that take advantage of both linguistic and visual features; this was the case in this study, where in both experimental conditions the story was presented with both verbal and visual information. Geise and Baden (2015) proposed a model of multimodal frame processing that integrates textual, verbal, and visual aspects, where meaning is extracted from complex stimuli, independently of their mode of presentation. According to these authors, 'all information obtained from visual, textual, or multimodal 
message ultimately feeds into the same kind of construction process wherein a coherent interpretation is formed' (p. 47). These considerations are consistent with the present findings, where the content of the story (presented mostly verbally in one experimental condition and both verbally and visually in the other experimental condition) led to similar results.

Note that our conclusion that the medium is relatively unimportant is specific to literature on vicarious contact interventions conducted in educational contexts. In fact, the written format may differ from the video format on many aspects. The former is characterized by verbal content while the latter is characterized by both verbal and visual content that may have additive or divergent effects depending on their consistency (Scheufele, 2004). In our study, consistent with previous literature, the written story was characterized by both visual content (e.g., in the written story there were images of characters) and verbal content (which was similar across mediums). Also note that children were presented with verbal content from an external source not only in the video but also in the reading story condition, since the stories were read-and shown-by researchers. However, we did not independently manipulate specific features between mediums (e.g., verbal or visual content), as this was not the aim of our study. Therefore, findings do not allow more generalized conclusions regarding whether the content is independent from the medium. Rather, they show that the effect of vicarious contact interventions, as they are typically conducted in educational contexts (Cameron \& Turner, 2017; Di Bernardo et al., 2017), does not differ as a function of being realized via story reading or video watching.

We are aware of two vicarious contact studies that, like we did, included both story reading and video watching simultaneously. Vezzali, Stathi, Giovannini, Capozza, and Trifiletti (2015, Studies 2 and 3) conducted two correlational studies to test the effects of reading Harry Potter books on outgroup attitudes. In the first (Study 2), number of Harry Potter books read was associated with more positive attitudes towards gay people among adolescents who identified more strongly with Harry Potter; number of Harry Potter films watched did not have any effect. In the second (Study 3), the number of Harry Potter books read was associated with more positive attitudes towards refugees via perspectivetaking among individuals who identified less strongly with Voldemort (the negative character of the Harry Potter saga); number of Harry Potter films watched was associated with less perspective-taking and attitudes towards refugees (marginal effects); the latter effect was significant among those less identified with Voldemort. These results seem in contrast with our findings, since they point to differential effects of reading versus watching. However, it should be noted that in the research mentioned above the authors did not manipulate vicarious contact in accordance with the premises of contact theory. Even more importantly, although both books and movies concerned the Harry Potter saga, the content of the two media was different, with books allowing greater introspection and character development while movies being more centred on action. Based on these considerations, we believe these results are not comparable with the present findings, but reinforce the importance of proper comparisons of vicarious contact via story reading or video watching.

Research that originated from the initial formulation of the extended contact hypothesis (Wright et al., 1997) has clearly distinguished between extended and vicarious contact as two separate contact forms only since 2011 (Dovidio et al., 2011; Mazziotta et al., 2011). Concerning vicarious contact, vicarious contact via the media (based for instance on stories that appear in television, radio or books) has been considered equivalent to other operationalizations of vicarious contact (such as ad hoc short stories 
and videos created for experimental/intervention purposes). Vezzali and Stathi (2020) proposed that vicarious contact via the media (labelled 'media vicarious contact') should be distinguished from vicarious contact that is not via the media. In fact, although both 'standard' vicarious contact and media vicarious contact can be operationalized via story reading (e.g., books) or video watching (e.g., television series, movies), the two might be different in important ways. Specifically, stories presented in the media, because they are prepared by experts of communication and because of generally greater length, can be characterized by higher levels of involvement. Psychological processes depending on involvement like transportation (Green \& Brock, 2000) and experience-taking (Kaufman \& Libby, 2012) resemble media vicarious contact instead of standard vicarious contact. In the present study, despite the fact that participants reported liking the story, it is unlikely that participants engaged in processes such as transportation or experience-taking. Therefore, the results obtained in this study cannot be readily generalized to media vicarious contact.

In the introduction, we reviewed literature on vicarious contact via story reading or video watching, noting that while story reading has been mostly used in the field, video watching has mainly been utilized in the laboratory. We argue that this discrepancy might have more practical than conceptual implications. Creating written stories for use in the field is likely to be less complex than creating videos. In fact, videos require identifying actors, the stage, eventual authorizations (e.g., from actors' parents, in case of under-age actors), editing, cooperation of multiple parties to create the final product, etc. Since interventions may be realized in multiple sessions, several videos would be required, making it rather impractical. We believe, however, that practical limitations should be overcome, and studies are needed where the different contact forms are experimentally compared. Our study goes precisely in this direction.

We believe this study has important practical implications. We showed that a single session of vicarious contact can have effects on several outcome variables, representing a viable strategy for a generalized improvement of intergroup relations. Based on the findings, practitioners can decide which medium (written story or video) is to be used for a vicarious contact intervention, depending on organizational constraints or practical difficulties. To the extent that the content of the story is designed according to vicarious contact principles, the medium through which it will be presented is less important. To the best of our knowledge, this is the first study that allows such a conclusion.

We acknowledge some limitations. One limitation is that, in contrast with most part of literature concerning vicarious contact via story reading in the field, our intervention consisted of a single session. Nonetheless, it proved effective in improving attitudes towards the outgroup; therefore, we believe these results are comparable with the general literature. In any case, future interventions should use multiple sessions and assess outcomes with some significant delay post-manipulation. This will allow to test the longevity of the effects of vicarious contact interventions. A further limitation is that we only considered a target outgroup (children with disability) among elementary schoolchildren; therefore, results are not generalizable to other target outgroups or age groups. For example, research can further explore the effectiveness of video watching versus story reading in contexts of threat or prolonged conflict and directly test whether the effects of both methods and their underlying processes are comparable. A third limitation is that some of the measures had low reliabilities; therefore, findings should be interpreted with caution. Fourth, since we only relied on self-reports, we cannot exclude the presence of social desirability effects. Future studies should use implicit and/or behavioural measures in order to test the outcomes of vicarious contact interventions. 
Finally, to our knowledge, some of the measures used (e.g., perspective-taking, affective empathy) have only been used in the Italian context; future studies are needed in order to provide cross-cultural validity to these measures.

In conclusion, this study shows that vicarious contact interventions based on story reading or video watching are equally effective in improving outgroup attitudes. Based on the evidence that vicarious contact is a promising strategy for prejudice reduction, researchers can further compare the different modalities through which vicarious contact can be implemented in order to better understand potential underlying processes. This will allow to develop more effective, theory-driven interventions that improve intergroup relations.

\section{Acknowledgements}

We wish to thank the Institute Marconi (Casalmaggiore, Cremona) and its teachers for their help in the organization and realization of the study.

\section{Conflicts of interest}

All authors declare no conflict of interest.

\section{Data availability statement}

The data that support the findings of this study are available at the following link: https://osf.io/ kqtvm/?view_only=4e8d34e7062942da9ac10ab145e0f782.

\section{References}

Aboud, F. E. (2003). The formation of in-group favoritism and out-group prejudice in young children: Are they distinct attitudes? Developmental Psychology, 39(1), 48-60. https://doi.org/10.1037/ 0012-1649.39.1.48

Aron, A., Aron, E. N., \& Smollan, D. (1992). Inclusion of other in the self scale and the structure of interpersonal closeness. Journal of Personality and Social Psychology, 63(4), 596-612. https://doi.org/10.1037/0022-3514.63.4.596

Aronson, K. M., Stefanile, C., Matera, C., Nerini, A., Grisolaghi, J., Romani, G., . . Brown, R. (2016). Telling tales in school: Extended contact interventions in the classroom. Journal of Applied Social Psychology, 46(4), 229-241. https://doi.org/10.1111/jasp.12358

Bandura, A. (1997). Self-efficacy: The exercise of control. New York, NY: Freeman.

Bandura, A. (1999). Social cognitive theory of personality. In D. Cervone \& Y. Shoda (Eds.), The coherence of personality (pp. 185-241). New York, NY: Guilford Press.

Bandura, A. (2002). Social cognitive theory of mass communication. In J. Bryant \& D. Zillmann (Eds.), Media effects: Advances in theory and research (2nd ed.) (pp. 121-153). Hillsdale, MI: Erlbaum.

Birtel, M. D., Di Bernardo, G. A., Stathi, S., Crisp, R. J., Cadamuro, A., \& Vezzali, L. (2019). Imagining contact reduces prejudice in pre-school children. Social Development, 28(4), 1054-1073. https://doi.org/10.1111/sode.12374

Birtel, M. D., Vezzali, L., \& Stathi, S. (2017). Extended contact and affective factors: A review and suggestions for future research. Testing, Psychometrics, Methodology in Applied Psychology, 25(2), 215-238. https://doi.org/10.4473/TPM25.2.4

Brown, R., \& Paterson, J. (2016). Indirect contact and prejudice reduction: Limits and possibilities. Current Opinions in Psychology, 11(5), 20-24. https://doi.org/10.1016/j.copsyc.2016.03.005 
Cameron, L., \& Rutland, A. (2006). Extended contact through story reading in school: Reducing children's prejudice toward the disabled. Journal of Social Issues, 62(3), 469-488. https://doi. org/10.1111/j.1540-4560.2006.00469.x

Cameron, L., Rutland, A., \& Brown, R. (2007). Promoting children's positive intergroup attitudes towards stigmatized groups: Extended contact and multiple classification skills training. International Journal of Behavioral Development, 31(5), 454-466. https://doi.org/10.1177/ 0165025407081474

Cameron, L., Rutland, A., Brown, R., \& Douch, R. (2006). Changing children's intergroup attitudes toward refugees: Testing different models of extended contact. Child Development, 77(5), 1208-1219. https://doi.org/10.1111/j.1467-8624.2006.00929.x

Cameron, L., Rutland, A., Hossain, R., \& Petley, R. (2011). When and why does extended contact work? The role of high quality direct contact and group norms in the development of positive ethnic intergroup attitudes amongst children. Group Processes and Intergroup Relations, 14(2), 193-206. https://doi.org/10.1177/1368430210390535

Cameron, L., \& Turner, R. N. (2017). Intergroup contact among children. In L. Vezzali \& S. Stathi (Eds.), Intergroup contact theory: Recent developments and future directions (pp. 151-168). Abingdon, UK: Routledge.

Capozza, D., Trifiletti, E., Vezzali, L., \& Favara, I. (2013). Can contact improve humanity attributions? International Journal of Psychology, 48(4), 527-541. https://doi.org/10.1080/00207594. 2012.688132

Castelli, L., Carraro, L., Pavan, G., Morelli, E., \& Carraro, A. (2012). The power of the unsaid: The influence of nonverbal cues on implicit attitudes. Journal of Applied Social Psychology, 42(6), 1376-1393. https://doi.org/10.1111/j.1559-1816.2012.00903.x

Cernat, V. (2011). Extended contract effects: Is exposure to positive outgroup exemplars sufficient or is interaction with ingroup members necessary? Journal of Social Psychology, 151(6), 737753. https://doi.org/10.1080/00224545.2010.522622

Cumming, G. (2009). Inference by eye: Reading the overlap of independent confidence intervals. Statistics in Medicine, 28(2), 205-220. https://doi.org/10.1002/sim.3471

Di Bernardo, G. A., Vezzali, L., Stathi, S., Cadamuro, A., \& Cortesi, L. (2017). Vicarious, extended and imagined intergroup contact: A review of interventions based on indirect contact strategies applied in educational settings. Testing, Psychometrics, Methodology in Applied Psychology, 24(1), 3-21. https://doi.org/10.4473/TPM24.1.1

Dovidio, J. F., Eller, A., \& Hewstone, M. (2011). Improving intergroup relations through direct, extended and other forms of in direct contact. Group Processes and Intergroup Relations, 14(2), 147-160. https://doi.org/10.1177/1368430210390555

Doyle, A. B., \& Aboud, F. E. (1995). A longitudinal study of white children: Racial prejudice as a social-cognitive development. Merrill-Palmer Quarterly, 41(2), 209-228.

Eller, A., Gómez, A., Vázquez, A., \& Fernández, S. (2017). Collateral damage for ingroup members having outgroup friends: Effects of normative versus counternormative interactions with an outgroup. Group Processes and Intergroup Relations, 20(4), 483-500. https://doi.org/10. 1177/1368430215612222

Esses, V. M., \& Dovidio, J. F. (2002). The role of emotions in determining willingness to engage in intergroup contact. Personality and Social Psychological Bulletin, 28(9), 1202-1214. https:// doi.org/10.1177/01461672022812006

Geise, S., \& Baden, C. (2015). Putting the image back into the frame: Modeling the linkage between visual communication and frame-processing theory. Communication Theory, 25(1), 46-69. https://doi.org/10.1111/comt.12048

Godin, G., \& Kok, G. (1996). The theory of planned behaviour: A review of its applications to health related behaviors. American Journal of Health Promotion, 11(2), 87-98. https://doi.org/10. 4278/0890-1171-11.2.87

Gómez, A., \& Huici, C. (2008). Vicarious intergroup contact and role of authorities in prejudice reduction. The Spanish Journal of Psychology, 11(1), 103-114. https://doi.org/10.1017/ S1138741600004169 
Gómez, A., Tropp, L. R., \& Fernández, S. (2011). When extended contact opens the door to future contact: Testing the effects of extended contact on attitudes and intergroup expectancies in majority and minority groups. Group Processes and Intergroup Relations, 14(2), 161-173. https://doi.org/10.1177/1368430210391119

Green, M. C., \& Brock, T. C. (2000). The role of transportation in the persuasiveness of public narratives. Journal of Personality and Social Psychology, 79(5), 701-721. https://doi.org/10. 1037/0022-3514.79.5.701

Greenwood, K., Carroll, C., Crowter, L., Jamieson, K., Ferraresi, L., Jones, A.-M., \& Brown, R. (2016). Early intervention for stigma towards mental illness? Promoting positive attitudes towards severe mental illness in primary school children. Journal of Public Mental Health, 15(4), 188199. https://doi.org/10.1108/JPMH-02-2016-0008

Hayes, A. F. (2013). Introduction to mediation, moderation, and conditional process analysis: A regression-based approach. New York, NY: Guilford Press.

Hodson, G., \& Hewstone, M. (Eds.) (2013). Advances in intergroup contact. New York, NY: Psychology Press.

Husnu, S., \& Crisp, R. J. (2010). Elaboration enhances the imagined contact effect. Journal of Experimental Social Psychology, 46(6), 943-950. https://doi.org/10.1016/j.jesp.2010.05.014

Husnu, S., Mertan, B., \& Cicek, O. (2018). Reducing Turkish Cypriots children's prejudice toward Greek Cypriots: Vicarious and extended intergroup contact through storytelling. Group Processes and Intergroup Relations, 21(1), 178-192. https://doi.org/10.1177/ 1368430216656469

Kaufman, G. F., \& Libby, L. K. (2012). Changing beliefs and behavior through experience-taking. Journal of Personality and Social Psychology, 103(1), 1-19. https://doi.org/10.1037/ a0027525

Kress, G., \& van Leeuwen, T. (2010). Reading images. The grammar ofvisual design. London, New York: Routledge.

Liebkind, K., Mähönen, T. A., Solares, E., Solheim, E., \& Jasinskaja-Lathi, I. (2014). Prejudicereduction in culturally mixed classrooms: The development and assessment of a theory-driven intervention among majority and minority youth in Finland. Journal of Community and Applied Social Psychology, 24(4), 325-339. https://doi.org/10.1002/casp.2168

Liebkind, K., Mäkinen, V., Jasinskaja-Lahti, I., Renvik, T. A., \& Solheim, E. F. (2019). Improving outgroup attitudes in schools: First step toward a teacher-led vicarious contact intervention. Scandinavian Journal of Psychology, 60(1), 77-86. https://doi.org/10.1111/sjop.12505

Liebkind, K., \& McAlister, A. L. (1999). Extended contact through peer modelling to promote tolerance in Finland. European Journal of Social Psychology, 29(5-6), 765-780. https://doi. org/10.1002/(SICI)1099-0992(199908/09)29:5/6<765:AID-EJSP958>3.0.CO;2-J

Lolliot, S., Fell, B., Schmid, K., Woelfer, R., Swart, H., Voci, A., Christ, O., New, R., \& Hewstone, M. (2015). Measures of intergroup contact. In G. Boyle, D. H. Saklofske \& G. Matthews (Eds.), Measures of personality and social psychological constructs (pp. 652-683). San Diego, CA: Academic Press.

Mäkinen, V., Liebkind, K., Jasinskaja-Lahti, I., \& Renvik, T. A. (2019). A teacher-led vicarious contact intervention in culturally mixed classrooms with in- and outgroup role models of intergroup friendship. Journal of School Psychology, 75, 27-40. https://doi.org/10.1016/j.jsp.2019.07.002

Mallett, R. K., \& Wilson, T. D. (2010). Increasing positive intergroup contact. Journal of Experimental Social Psychology, 46(2), 382-387. https://doi.org/10.1016/j.jesp.2009.11.006

Mazziotta, A., Mummendey, A., \& Wright, S. C. (2011). Vicarious intergroup contact effects: Applying social-cognitive theory to intergroup contact research. Group Processes and Intergroup Relations, 14(2), 255-274. https://doi.org/10.1177/1368430210390533

McKeown, S., Williams, A., \& Pauker, K. (2017). Stories that move them: Changing children's behaviour toward diverse peers. Journal of Community and Applied Social Psychology, 27(5), 381-387. https://doi.org/10.1002/casp.2316

Nelson, T. E., Oxley, Z. M., \& Clawson, R. A. (1997). Toward a psychology of framing effects. Political Behavior, 19(3), 221-246. https://doi.org/10.1023/A:1024834831093 
Nguyen, E., Chen, T. F., \& O'Reilly, C. L. (2012). Evaluating the impact of direct and indirect contact on the mental health stigma of pharmacy students. Social Psychiatry and Psychiatric Epidemiology, 47(7), 1087-1098. https://doi.org/10.1007/s00127-011-0413-5

Pagotto, L., \& Voci, A. (2013). Direct and mass-mediated contact: The role of different intergroup emotions. Testing, Psychometrics, methodology in Applied Psychology, 20(4), 365-381. https://doi.org/10.4473/TPM20.4.5

Pettigrew, T. F., \& Tropp, L. R. (2006). A meta-analytic test of intergroup contact theory. Journal of Personality and Social Psychology, 90(5), 751-783. https://doi.org/10.1037/0022-3514.90.5. 751

Pettigrew, T. F., \& Tropp, L. R. (2008). How does intergroup contact reduce prejudice? Meta-analytic tests of three mediators. European Journal of Social Psychology, 38(6), 922-934. https://doi. org/10.1002/ejsp.504

Petty, R. E., \& Cacioppo, J. T. (1986). Communication and persuasion: Central and peripheral routes to attitude change. New York, NY: Springer-Verlag.

Price, V., Tewksbury, D., \& Powers, E. (1997). Switching trains of thought: The impact of news frames on readers' cognitive responses. Communication Research, 24(5), 481-506. https:// doi.org/10.1177/009365097024005002

Scheufele, B. (2004). Framing-effects approach: A theoretical and methodological critique. Communications, 29(4), 421-428. https://doi.org/10.1515/comm.2004.29.4.401

Schrijvers, M., Jannsen, T., Fialho, O., \& Rijlaarsdam, G. (2019). Gaining insight into human nature: A review of literature classroom intervention studies. Review of Educational Research, 89(1), 345. https://doi.org/10.3102/0034654318812914

Sedikides, C., Olsen, N., \& Reis, H. T. (1993). Relationships as natural categories. Journal of Personality and Social Psychology, 64(1), 71-82. https://doi.org/10.1037/0022-3514.64.1.71

Shelton, J. N., \& Richeson, J. A. (2005). Intergroup contact and pluralistic ignorance. Journal of Personality and Social Psychology, 88(1), 91-107. https://doi.org/10.1037/0022-3514.88.1.91

Stephan, W. G., \& Stephan, C. W. (1985). Intergroup anxiety. Journal of Social Issues, 41(1), 157175. https://doi.org/10.1111/j.1540-4560.1985.tb01134.x

Taylor, L. K., \& Glen, C. (2020). From empathy to action: Can enhancing host-society children's empathy promote positive attitudes and prosocial behaviour toward refugees? Journal of Community and Applied Social Psychology, 30(2), 214-226. https://doi.org/10.1002/casp. 2438

Turner, R. N., Hewstone, M., Voci, A., Paolini, S., \& Christ, O. (2007). Reducing prejudice via direct and extended cross-group friendship. European Review of Social Psychology, 18(1), 212-255. https://doi.org/10.1080/10463280701680297

Turner, R. N., Hewstone, M., Voci, A., \& Vonofakou, C. (2008). A test of the extended contact hypothesis: The mediating role of intergroup anxiety, perceived ingroup and outgroup norms, and inclusion of the outgroup in the self. Journal of Personality and Social Psychology, 95(4), 843-860. https://doi.org/10.1037/a0011434

Vezzali, L., Birtel, M. D., Di Bernardo, G. A., Stathi, S., Crisp, R. J., \& Cadamuro, A. (2019). Don't hurt my outgroup friend: Imagined contact promotes intentions to counteract bullying. Group Processes and Intergroup Relations, https://doi.org/10.1177/1368430219852404

Vezzali, L., Di Bernardo, G. A., Stathi, S., Cadamuro, A., Lasticova, B., \& Andraščiková, S. (2018). Secondary transfer effect among children: The role of social dominance orientation and outgroup attitudes. British Journal of Social Psychology, 57(3), 547-566. https://doi.org/10. $1111 /$ bjso. 12248

Vezzali, L., Di Bernardo, G. A., Stathi, S., Visintin, E. P., \& Hewstone, M. (2019). Using intercultural videos of direct contact to implement vicarious contact: A school-based intervention that improves intergroup attitudes. Group Processes and Intergroup Relations, 22(7), 1059-1076. https://doi.org/10.1177/1368430218809885

Vezzali, L., Hewstone, M., Capozza, D., Giovannini, D., \& Wölfer, R. (2014). Improving intergroup relations with extended and vicarious forms of indirect contact. European Review of Social Psychology, 25(1), 314-389. https://doi.org/10.1080/10463283.2014.982948 
Vezzali, L., Stathi, S., Crisp, R. J., Giovannini, D., Capozza, D., \& Gaertner, S. L. (2015). Imagined intergroup contact and common ingroup identity: An integrative approach. Social Psychology, 46(5), 265-276. https://doi.org/10.1027/1864-9335/a000242

Vezzali, L., Stathi, S., \& Giovannini, D. (2012). Indirect contact through book reading: Improving adolescents' attitudes and behavioral intentions toward immigrants. Psychology in the Schools, 49(2), 148-162. https://doi.org/10.1002/pits.20621

Vezzali, L., Stathi, S., Giovannini, D., Capozza, D., \& Trifiletti, E. (2015). The greatest magic of Harry Potter: Reducing prejudice. Journal of Applied Social Psychology, 45(2), 105-121. https://doi. org/10.1111/jasp.12279

Vezzali, L., Stathi, S., Giovannini, D., Capozza, D., \& Visintin, E. P. (2015). "And the best essay is. ..”: Extended contact and cross-group friendships at school. British Journal of Social Psychology, 54(4), 601-615. https://doi.org/10.1111/bjso.12110

Vezzali, L., \& Stathi, S. (2020). Towards a better world: Using intergroup contact to fight prejudice and negative attitudes, London, UK: Routledge.

West, K., \& Turner, R. N. (2014). Using extended contact to improve physiological responses and behaviour toward people with schizophrenia. Journal of Experimental Social Psychology, 50, 57-64. https://doi.org/10.1016/j.jesp.2013.06.009

Wright, S. C., Aron, A., McLaughlin-Volpe, T., \& Ropp, S. A. (1997). The extended contact effect: Knowledge of cross-group friendships and prejudice. Journal of Personality and Social Psychology, 73(1), 73-90. https://doi.org/10.1037/0022-3514.73.1.73

Received 26 February 2020; revised version received II June 2020 